\title{
Verification of the Control System Performance using Viability Theory
}

\author{
Majid Ghaniee Zarch \\ Department of Electrical Engineering \\ Iran University of Science and \\ Technology (IUST), Narmak, \\ 16846 Tehran, Iran \\ Email: majidghaniee@iust.ac.ir
}

\author{
Vicenç Puig \\ Institut de Robtica i Informtica \\ Industrial (CSIC-UPC). Carrer Llorens \\ Artigas, 4-6, 08028 Barcelona \\ Email: vicenc.puig@upc.edu
}

\author{
Javad Poshtan \\ Department of Electrical Engineering \\ Iran University of Science and \\ Technology (IUST), Narmak, \\ 16846 Tehran, Iran \\ Email: jposhtan@iust.ac.ir
}

\begin{abstract}
The development of efficient methods for the control system performance verification has drawn a lot of attention recently. In this paper, the use of viability theory for this purpose is investigated in case of non-linear systems. In particular, verification algorithms based on the use of the computation of invariance and viability kernels and capture basin are proposed. A Lagrangian method has been used in order to approximate these sets for nonlinear systems. Because of simplicity and efficient computations, zonotopes are adopted for set representation. An application example based on a well known control benchmark is provided in order to show the effectiveness of the proposed method.
\end{abstract}

\section{INTRODUCTION}

Nowadays, verification of the performance of a designed control loop has drawn a lot of attention. Reachability analysis is widely used in this area as an acceptable tool for verification [1]. Safety of the system is a major issue that is investigated using reachability analysis [2]. There is also a rich literature in experimental verification of control systems [3], [4], [5].

In assessing the control performance, the goal is essentially to determine whether changes have occurred in some measure or characteristic parameter of the system [6]. Let the true process parameter of interest be denoted by $\theta$. This might be the process states, variance or any other performance indices. To detect a change in $\theta$, it is common to test a null hypothesis:

$$
H_{0}: \theta=\theta_{0}
$$

where $\theta_{0}$ is the desired process characteristics. Testing the null hypothesis requires specification of other parameters, such as critical values. When the test statistics are calculated sequentially, the performance of the test procedure is often characterised in terms of its run length distribution [7].

Viability theory provides a solid framework for control synthesis of constrained dynamical systems in a set-valued form [8], [9], and has been utilized in many applications such as robotics [10], aircraft collision avoidance [11] and air traffic management [12]. It also plays an important role in safety verification in control systems, a particular important problem for high-risk, expensive, or safety-critical applications. In many engineering systems, input constraints limit the systems ability to remain within a desired safe region of operation. For such systems, constraints on the state space determine the safe set. It is important to identify the subset of the safe set for which the existence of a control input that keeps the states of the system within the safe region can be guaranteed.

In this paper, three concepts in viability theory are used to verify performance of the control system. These concepts are invariance kernel, viability kernel and capture basin. The main contribution of this paper is an algorithm proposed for performance verification of a control system using these definitions from viability theory. This algorithm is developed based on the equivalency between concepts that until now were used for performance verification and viability theory concepts. The difficulty with viability theory relies on kernel computations. This paper also proposes algorithms to find these kernels. Because of simplicity and efficient computations, we adopt zonotopes for set representation, while for system representation the non-linear system model is brought to a Linear Parameter Varying (LPV) representation. The representation of the non-linear model in this way facilitates the set computations based on zonotopes that are required to apply the viability theory to the control system performance verification problem.

This paper is organized as follows. In Section II, some preliminaries about viability theory concepts are provided. In Section III, we show how viability theory sets can be calculated using LPV nonlinear system representation. The algorithm for performance verification is provided in Section IV. Simulation results are provided in Section V. In this Section, an example on two-tank system is given in order to illustrate the proposed method. Finally, concluding remarks are provided in Section VI.

\section{PRELIMINARY CONCEPTS}

In control engineering, a state space representation is a mathematical model of a physical system as a set of inputs $u$, state variables $x$ and unknown inputs $w$, related by a set dynamic equations represented by

$$
\left\{\begin{array}{l}
\mathscr{L}(x(t))=f(x(t), u(t), w(t)) \\
x(t) \in X \\
u(t) \in U \\
w(t) \in W
\end{array}\right.
$$

where the time $t$ ranges throughout a time domain $T$. The time domain $T$ can be either continuous or discrete. $\mathscr{L}$ is the differential operator corresponding to the given time domain 
(differentiation in the case of a continuous-time system and differencing in the case of a discrete-time system). It is assumed that the above system is defined in a proper open set $\mathrm{O} \subseteq \mathbb{R}^{n}$ and that there exist a globally defined solution for every initial condition $x(0) \in \mathrm{O}$. The evolutionary system:

$$
S: X \rightarrow \mathbb{C}(0,+\infty ; X)
$$

maps any initial state $x \in X$ to the set $S(x)$ of evolutions $x($. starting from $x(0)$ and governed by (1).

Viability theory is concerned with ensuring that a system state remains within a viability constraint set $K \subseteq \mathbb{R}^{d}$. Any trajectory of system (1) that leaves the set $K$ at some point in time is considered to be no longer viable.

Definition 2.1 (Viability Kernel): The viability kernel of $K$ under the evolutionary system $S$ is the set $\operatorname{Viab}_{S}(k)$ of initial states $x(0) \in K$ from which starts at least one evolution $x(t) \in$ $S(x)$ viable in $K$ for all times $t \geqslant 0$ :

$$
\operatorname{Viab}_{S}(K) \triangleq\left\{\begin{array}{l}
x(0) \in K \mid \exists x(.) \in S(x) \\
\text { such that } \forall t \geqslant 0, x(t) \in K
\end{array}\right\}
$$

Sometimes, from the engineering point of view, the existence of at least one solution in $S$ is not enough, since nothing is said about all other possible solutions. So, another stronger concept is defined known as the invariance kernel.

Definition 2.2 (Invariance Kernel): Let $K \subset X$ be an environment and $C \subset K$ be a target. The subset $\operatorname{Inv}_{S}(K, C)$ of initial states $x(0) \in K$ such that all evolutions $x(t) \in S(x)$ starting at $x(0)$ are viable in $K$ for all $t \geqslant 0$ or viable in $K$ until they reach $C$ in finite time is called the invariance kernel of $K$ with target $C$ under $S$.

$$
\operatorname{Inv}_{S}(K) \triangleq\{x(0) \in K \mid \forall x(.) \in S(x), \forall t \geqslant 0, x(t) \in K\}
$$

Positive invariance in set theory has the same definition as invariance kernel. Viability kernel and weak positive invariance are also equivalent definitions in viability and set theories, respectively [9]. Capture basin is another concept that has a wide range of applications, for example, in process control [13] and economics [14].

Definition 2.3 (Capture Basin): The capture basin of $C$ (viable in $K$ ) under the evolutionary system $S$ is the set $\operatorname{Capt}_{S}(K, C)$ of initial states $x(0) \in K$ from which starts at least one evolution $x(t) \in S(x)$ viable in $K$ on $[0, T)$ until the finite time $T$ when the evolution reaches the target at $x(T) \in C$.

Reachability analysis identifies the set of states backward (forward) reachable by a constrained dynamical system from a given target (initial) set of states. The notions of maximal and minimal reachability analysis were introduced in [15].

Definition 2.4 (Forward Maximal Reachable Set): The forward maximal reachable set at time instant $k$ is the set of states for which there exists an input such that the trajectories emanating from initial states in $T$ reach that set exactly at time instant $t$ :

$$
\operatorname{Reach}_{t}^{F}(T) \triangleq\left\{x(t) \in \mathbb{R}^{n} \mid \exists u(.) \in U_{[0, t]}, x(0) \in T\right\}
$$

Definition 2.5 (Backward Maximal Reachable Set): The backward maximal reachable set at time instant $t$ is the set

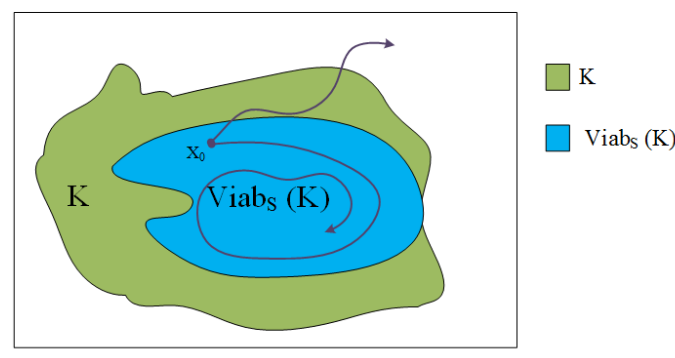

(a)

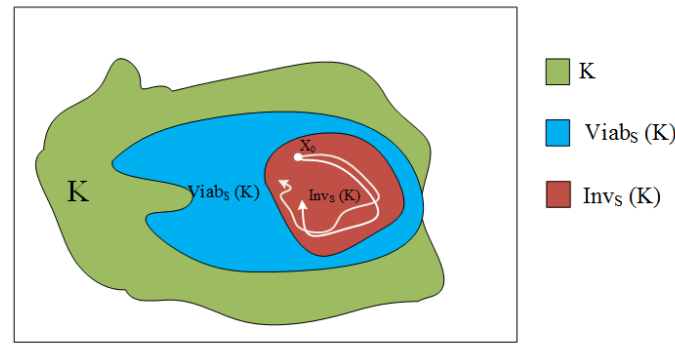

(b)

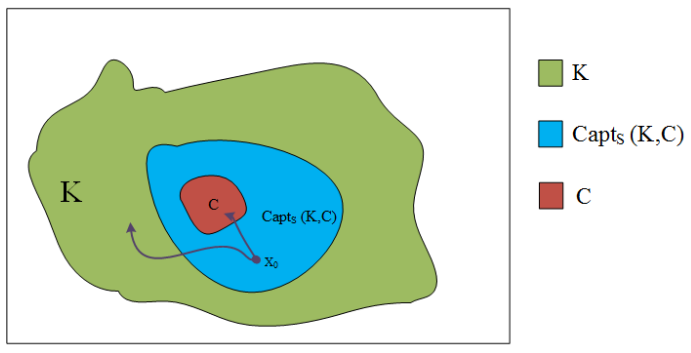

(c)

Fig. 1. A sample of: (a) viability kernel (b) invariance kernel (c) capture basin

of initial states for which there exists an input such that the trajectories emanating from those states reach $T$ exactly at time instant $t$ :

$$
\operatorname{Reach}_{t}^{B}(T) \triangleq\left\{x_{0} \in \mathbb{R}^{n} \mid \exists u(.) \in U_{[0, t]}, x(t) \in T\right\}
$$

\section{Computation OF Viability SEts}

The difficulty with the application of viability theory is the computation of the related sets presented in Section 2. In this section, several algorithms are proposed in order to derive these sets based on zonotopic sets and the LPV representation of the non-linear system. Here, we use the discrete-time quasi-LPV model on the non-linear system (1):

$$
x(t+1)=A(\rho(t)) x(t)+B(\rho(t)) u(t)+E(\rho(t)) w(t)
$$

where $x(t) \in X$ is the state, $u(t) \in U$ are the control inputs and $w(t) \in W$ are the unknown inputs.

\section{A. Invariance Kernel Computation}

Using Lemma 4.1, the zonotope $X_{t}$ that bounds the trajectories of the system (6) at instant $t$ is computed from 
the previous approximating zonotope at time instant $t-1$, $X_{t-1}=x_{t-1}^{c} \oplus H_{t-1}^{\bar{x}} \beta^{n}$

$$
\operatorname{Reach}_{1}^{F}\left(X_{t-1}\right)=X_{t}=x_{t}^{c} \oplus H_{t}^{\bar{x}} \beta^{n}
$$

where

$$
\begin{aligned}
& x_{t}^{c}=\operatorname{mid}(A(\rho(t-1))) x_{t-1}^{c}+\operatorname{mid}(B(\rho(t-1))) u^{c} \\
& +\operatorname{mid}(E(\rho(t-1))) w^{c} \\
& H_{t}^{\bar{x}}=\left[\begin{array}{llllll}
J_{1} & J_{2} & J_{3} & J_{4} & J_{5} & J_{6}
\end{array}\right] \\
& J_{1}=\operatorname{seg}\left(\diamond A(\rho(t-1)) H_{t-1}^{\bar{x}}\right) \\
& J_{2}=\frac{\operatorname{diam}(A(\rho(t-1)))}{2} x_{t-1}^{c} \\
& J_{3}=\operatorname{seg}\left(\diamond B(\rho(t-1)) H^{\bar{u}}\right) \\
& J_{4}=\frac{\operatorname{diam}(B(\rho(t-1)))}{2} u^{c} \\
& J_{5}=\operatorname{seg}\left(\diamond E(\rho(t-1)) H^{\bar{w}}\right) \\
& J_{6}=\frac{\operatorname{diam}(E(\rho(t-1)))}{2} w^{c}
\end{aligned}
$$

where 'mid' denotes the center and 'diam' the diameter of the interval, $\diamond$ is zonotope inclusion and $\operatorname{seg}(Q)=H$ considering that $Q=\pi+H \beta^{r}$ is a zonotope [16]. Note that the set of states has an increasing number of segments when generating the zonotope $X_{t}$ using this method. In order to control the domain complexity, a reduction step have to be used. Here, we use the method proposed in [17] to reduce the zonotope complexity. It is also important to note that $\rho(t-1)$ must be calculated based on information from $x_{t-1}^{c}$.

The sequence of sets $\operatorname{Reach}_{1}^{F}\left(X_{t}\right)$ for the dynamic system (6) can be used iteratively to find invariance kernel as follows

$$
\operatorname{Inv}(X)=\bigoplus_{t=0}^{\infty} \operatorname{Reach}_{1}^{F}\left(X_{t}\right)
$$

An algorithm is proposed in order to calculate invariance kernel based on above discussion.

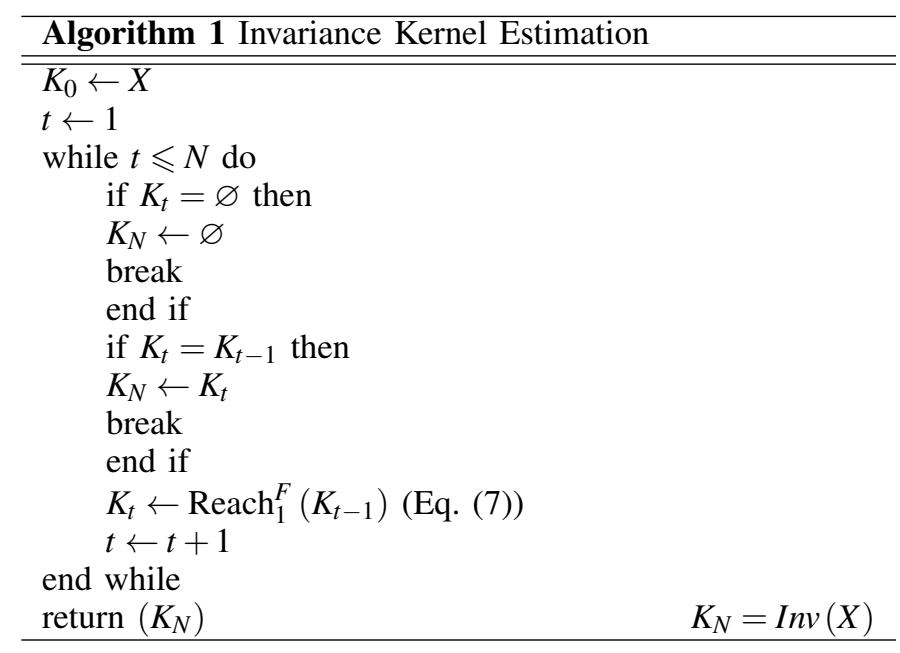

\section{B. Viability Kernel Computation}

Lagrangian methods have been successfully applied to the computation of reachable sets [18]. In contrast to Eulerian methods, Lagrangian methods use representations that follow the vector field flow. Since Lagrangian methods do not depend on gridding the state space, it is computationally feasible to analyze high-dimensional systems.

In this section, based on [19], a method of expressing finite horizon viability kernels in terms of reachable sets is presented. This provides a modified version of Saint-Pierres viability kernel algorithm that can be implemented using efficient and scalable techniques developed within the context of reachability analysis. We can reformulate this recursive definition of the finite horizon viability kernels $K_{n}$, in terms of the backward reach set over one discrete-time $\operatorname{step~} \operatorname{Reach}_{t}^{B}(X)$.

Theorem 1. The sequence of finite horizon viability kernels $K_{n}$ can be computed recursively in terms of reach sets as

$$
\begin{aligned}
& K_{0}=K \\
& K_{t+1}=K_{0} \cap \operatorname{Reach}_{1}^{B}\left(K_{t}\right)
\end{aligned}
$$

Proof. See [19].

Now, considering nonlinear system expressed in discretetime in the form (6), the backward reachable set over a single time step is computed as

$\operatorname{Reach}_{t}^{B}(X)=A(\rho(t))^{-1}\{X \oplus(-B(\rho(t))) U \oplus(-E(\rho(t))) W\}$

Here $A(.)^{-1}$ denotes the pre-image of a set under the map $A: \mathbb{R}^{d} \rightarrow \mathbb{R}^{d}$. We will assume that $A$ is non-singular, and thus the pre-image of $A$ can be calculated simply by applying the linear transformation $A(\rho(t))^{-1}$ to the set

$$
X \oplus(-B(\rho(t))) U \oplus(-E(\rho(t))) W
$$

Considering zonotopic sets, (8) can be rewritten in order to calculate backward reach set

$$
\begin{aligned}
& \operatorname{Reach}_{t}^{B}(X)=X_{t-1}=x_{t-1}^{c} \oplus H_{t-1}^{\bar{x}} \beta^{n} \\
& x_{t-1}^{c}=\operatorname{mid}\left(A(\rho(t))^{-1}\right) x_{t}^{c}+\operatorname{mid}\left(-A(\rho(t))^{-1} B(\rho(t))\right) u^{c} \\
& +\operatorname{mid}\left(-A(\rho(t))^{-1} E(\rho(t))\right) w^{c} \\
& H_{t-1}^{\bar{x}}=\left[\begin{array}{llllll}
J_{1} & J_{2} & J_{3} & J_{4} & J_{5} & J_{6}
\end{array}\right] \\
& J_{1}=\operatorname{seg}\left(\diamond A(\rho(t))^{-1} H_{t}^{\bar{x}}\right) \\
& J_{2}=\frac{\operatorname{diam}\left(A(\rho(t))^{-1}\right)}{2} x_{t}^{c} \\
& J_{3}=\operatorname{seg}\left(\diamond-A(\rho(t))^{-1} B(\rho(t)) H^{\bar{u}}\right) \\
& J_{4}=\frac{\operatorname{diam}\left(-A(\rho(t))^{-1} B(\rho(t))\right)}{2} u^{c} \\
& J_{5}=\operatorname{seg}\left(\diamond-A(\rho(t))^{-1} E(\rho(t)) H^{\bar{w}}\right) \\
& J_{6}=\frac{\operatorname{diam}\left(-A(\rho(t))^{-1} E(\rho(t))\right)}{2} w^{c}
\end{aligned}
$$

This method is similar to the one that was proposed for invariance kernel computation. The difference is that in invariance kernel computation forward reachable set is used, but backward reachable set is used in viability kernel computation. Here, a similar algorithm for computing viability kernel for system (6) based on (9) and (11) is presented. 


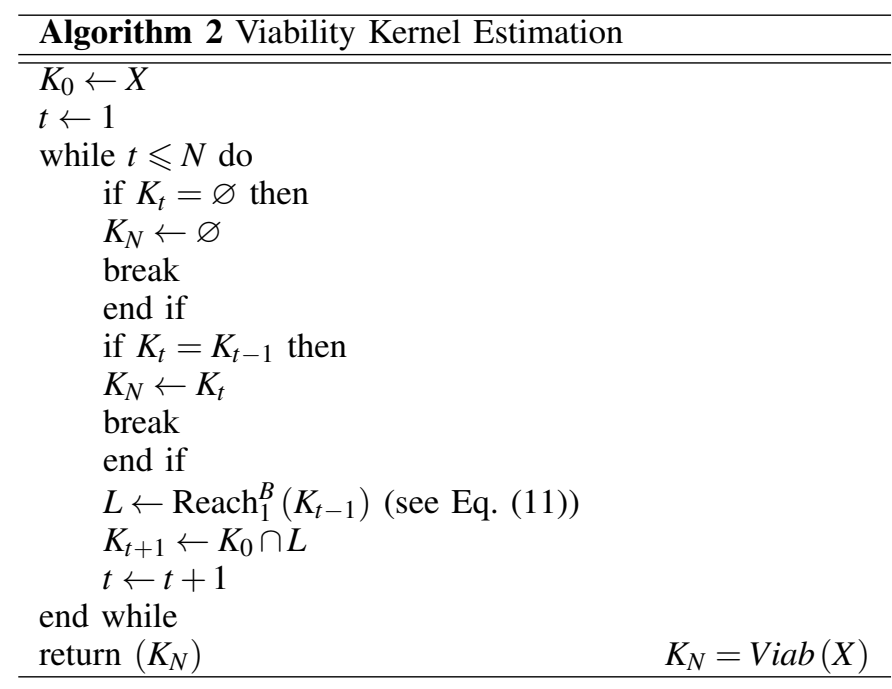

\section{Capture Basin Computation}

Based on capture basin definition, it is clear that we can easily modify Algorithm 2 in order to find capture basin. In the viability kernel definition, no time constraint is considered. Therefore, the algorithm is repeated until it converges to a set. But, in the capture basin concept, there is time limit that can be considered by a small change in stop criteria of the viability algorithm. Actually, we must find backward reachable tube for each time instant $t$. In this manner, Algorithm 3 is proposed.

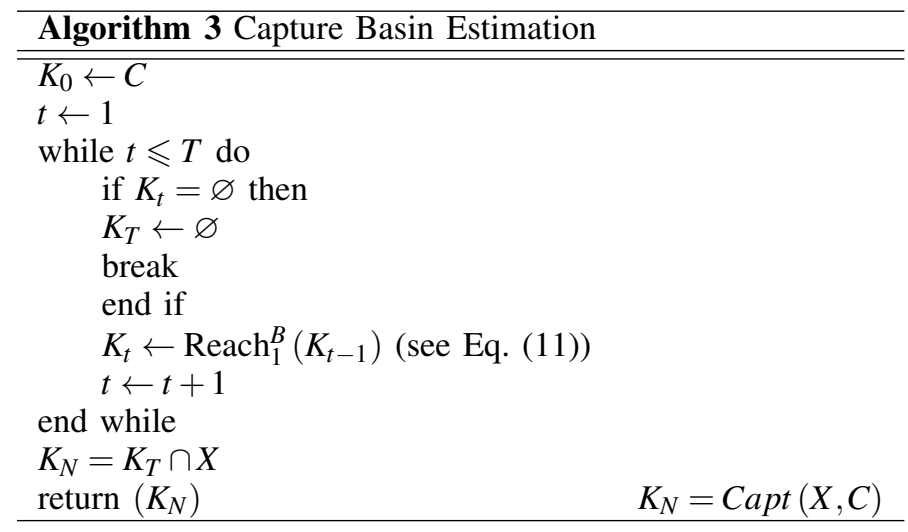

\section{SYSTEM VERIFICATION}

\section{A. Problem description}

The problem of system verification has recently received much attention (see e.g. [1]) providing the answer to questions as: is a potentially unsafe configuration, or state, reachable from an initial configuration?. The problem of system verification may be encoded as a condition on the region of operation in the system state space: given a region of the state space that represents unsafe operation, prove that the set of states from which the system can enter this unsafe region has empty intersection with the system initial states (see Fig. 2).

In this paper, the aim is to investigate if the performance requirements on the closed-loop system are satisfied. This can be illustrated by using the notion of the system behaviour (Fig. 3 ). The plant has the behaviour $\beta_{o}$ and the controller $\beta_{c}$. The set $\beta_{c}$ describes the I/O pairs $(U, Y)$ that satisfies the control law.

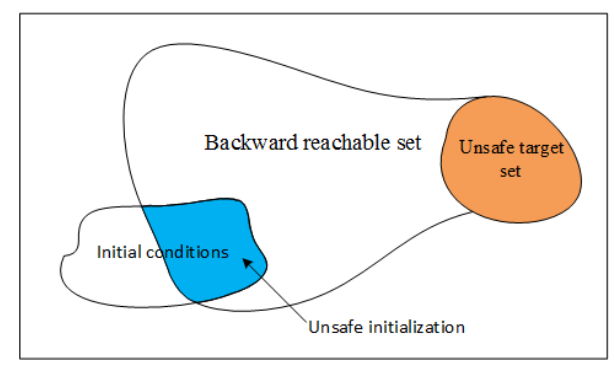

Fig. 2. Backward reachable set for safety verification

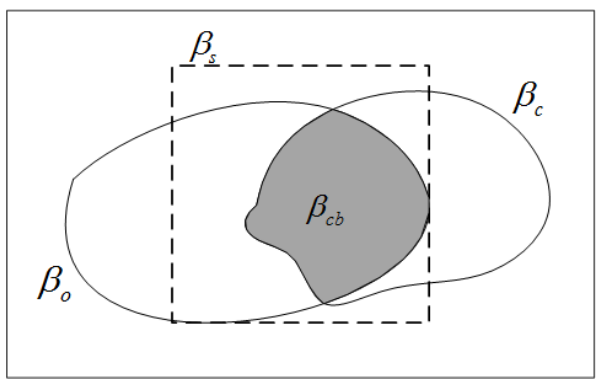

Fig. 3. System behaviour characterization

Since the I/O pairs of the closed loop system are consistent with both the plant and the controller, the behaviour of the closed-loop system is given by the intersection $\beta_{c b}=\beta_{o} \cap \beta_{c}$ (which is drawn in grey). The behavioural requirements of the system can be shown as the $\beta_{s}$ of those I/O pairs. As the grey set lies completely within the set $\beta_{s}$

$$
\beta_{c b} \subset \beta_{s}
$$

the closed-loop system satisfies the performance specifications.

\section{B. System verification using viability theory}

Based on the viability concepts recalled in Section II and the algorithms for computing the sets involved presented before, it can be readily deduced that there are some similarities that allow to use viability theory in performance verification. It is desirable to find equivalency between concepts that until now is used for performance monitoring and viability theory concepts. The system is required to work in a set that preserves the safety of the system. This set, that is shown with $\beta_{s}$ in the Fig. 3, can be represented with viability kernel, i.e:

$$
\beta_{s} \equiv \operatorname{Viab}_{S}(K)
$$

Therefore, if the system works in viability kernel, safety requirements are met. In the viability kernel definition, there is a limitation that the system must have at least one evolution that remains in the set. This is close to the concept of Lyapanov theory for stability.

For assessing and verifying if the controller performance is acceptable, capture basin is used. Capture basin is a set that shows the capability of the system to go toward a target set. After finding viability kernel based on constraints of states and inputs, the capture basin can be obtained. In this manner, it is possible to consider viability kernel or a part of the set (based on steady state or a predefined objective trajectory) as the target when determining the capture basin. For the equivalency 
purpose, the intersection of the system and controller behaviour set can be considered as the capture basin:

$$
\beta_{c b} \equiv \operatorname{Capt}_{S}(K, C)
$$

This means that if the system works in the capture basin, it has the capability to arrive to the target in a finite and desired time that is used in the capture basin derivation algorithm. The target can be considered as small set near steady state inside viability kernel or a small set around a predefined trajectory. Also, the invariance kernel can be used as target in the capture basin computation:

$$
C \equiv \operatorname{Inv}_{S}(K)
$$

\section{Illustrative EXAMPLE}

In this section, the algorithm for system verification developed in the previous sections is applied to a coupled-tank process that is modeled in a quasi LPV form.

The dynamic model of the water tank system can be written as [20]

$$
\begin{aligned}
& \dot{h}_{1}(t)=-(s / S) \sqrt{2 g} \sqrt{h_{1}(t)}+(\kappa / S) u(t)+w_{1}(t) \\
& \dot{h}_{2}(t)=(s / S) \sqrt{2 g}\left[\sqrt{h_{1}(t)}-\sqrt{h_{2}(t)}\right]+w_{2}(t)
\end{aligned}
$$

where $u(t)$ is the voltage applied to the pump, $h_{1}(t)$ and $h_{2}(t)$ are system states, $w_{1}(t), w_{2}(t)$ are bounded state perturbation and the parameters are as follows: $S=15.5179 \mathrm{~cm}^{2}$ is the cross section area of the tanks; $s=0.1781 \mathrm{~cm}^{2}$ the cross section of the tanks outflow orifice; $\kappa=3.3 \mathrm{~cm}^{3} / V s$ is the gain of the pump; and $g=981 \mathrm{~cm} / \mathrm{s}^{2}$ the gravitational constant. After Euler discretisation with sampling period $\tau=1 s$, the whole system is formulated in its quasi-LPV form through parameter non-linear embbeding approach [20]

$$
\begin{aligned}
& x(t+1)=A(\rho(t)) x(t)+B u(t)+E w(t) \\
& y(t)=C x(t)+\eta(t)
\end{aligned}
$$

where

$$
\begin{aligned}
& x(t)=\left[\begin{array}{ll}
h_{1}(t) & h_{2}(t)
\end{array}\right]^{T} \\
& A(\rho(t))=I+\tau\left[\begin{array}{cc}
-\rho_{1}(t) & 0 \\
\rho_{1}(t) & -\rho_{2}(t)
\end{array}\right] \\
& B=\tau\left[\begin{array}{ll}
\kappa / S & 0
\end{array}\right]^{T} \\
& C=\left[\begin{array}{ll}
0 & 1
\end{array}\right] \\
& E=\tau\left[\begin{array}{ll}
1 & 1
\end{array}\right]^{T} \\
& \eta(t)=\left[\begin{array}{ll}
\eta_{1}(t) & \eta_{2}(t)
\end{array}\right]^{T}
\end{aligned}
$$

The varying parameters are defined as follows

$$
\rho_{i}(t)=\frac{s}{S} \sqrt{\frac{2 g}{h_{i}(t)}}, \quad i=1,2
$$

$\eta(t)$ is measurement noise matrix. Disturbances and noises are considered bounded by means of zonotopes with center in 0 and segment of 0.01 . Let assume that the system should satisfy the following specifications:

- the goal is for $h_{2}(t)$ to track a reference of $5 \mathrm{~cm}$.

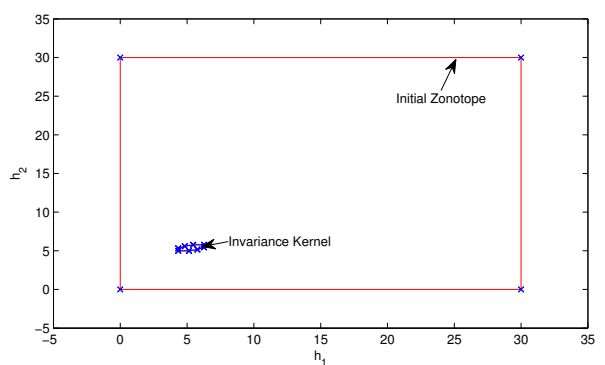

Fig. 4. Invariance kernel of two tank system

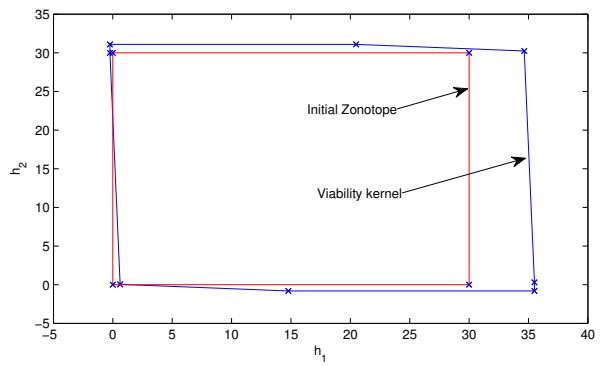

Fig. 5. Viability kernel of two tank system

- $\quad$ it must have the ability to go near the reference in 5 sample times.

- $\quad$ the system must work in a safe area.

For finding viability and invariance kernels, initial zonotope for states $x$ is considered as:

$$
X_{0}=\left[\begin{array}{l}
15 \\
15
\end{array}\right] \oplus\left[\begin{array}{cc}
15 & 0 \\
0 & 15
\end{array}\right] \beta^{2}
$$

The initial zontope for states is chosen to cover all the possible values for states. The zonotope for the input is chosen in order to achieve the reference. Therefore, for finding invariance kernel (and capture basin), it is considered as:

$$
U=5.5 \oplus 1 \beta
$$

and for finding viability kernel:

$$
U=0 \oplus 1 \beta
$$

Invariance and viability kernels of this system are computed using Algorithms 1 and 2, respectively. They are shown in Figures 4 and 5. Invariance kernel is around the steady state of the system and can be used as a tool for detecting faults in steady state [20]. Also, it can be used as initial set for capture basin construction. Viability kernel can be interpreted as a tool for verifying if the system has the ability to reach the steady state. In this example, because in each height there is a possibility to go to desired steady state, viability kernel covers all the initial zonotope. It means that if no time limitation is considered, each state can reach the steady state. Hence, system is working in safe area (viability kernel).

Capture basin is computed by means of Algorithm 3 using initial zonotope (invariance kernel): 


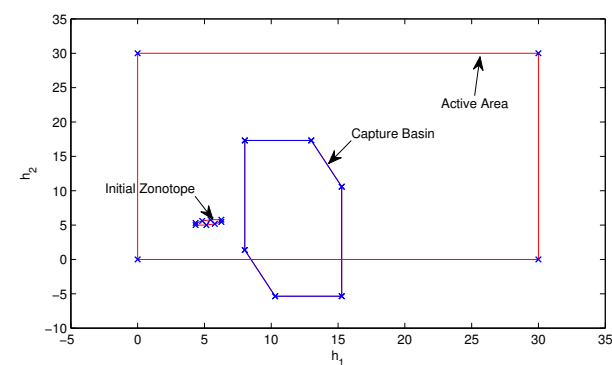

Fig. 6. Capture basin of two tank system in 5 steps

$$
Z_{0}=\left[\begin{array}{l}
0.41 \\
0.68
\end{array}\right] \oplus\left[\begin{array}{cccc}
0.41 & 0.4 & 0.04 & 0 \\
0.54 & 0.18 & 0 & 0.33
\end{array}\right] \beta^{2}
$$

for constructing capture basin, five time steps are considered. Regarding sampling time of the $\operatorname{system}(\tau=1 s)$, the system requires 5 seconds to reach the steady state (initial zonotope). Capture basin is the intersection of active area and computed capture basin. Using the capture basin, it can be assessed if the system has the possibility to achieve the desired set. It must be pointed out that starting inside capture basin do not mean that the system will inevitably go to the target in 5 seconds. This means that the system has the opportunity to go there in desired time.

\section{CONCLUSION}

In this paper, viability theory is used to verify performance of a control system. In this manner, a systematic way for evaluating performance of a control system is provided. The paper also proposes a set of algorithms based on the use of zonotopes and the LPV representation of the system for computing the viability theory sets. An algorithm that allows the system performance verification using these sets is also proposed. An example based on a tank system is provided to illustrate the proposed approach. In viability theory, there are more general definitions like absorption basin, restoring viability and so on that can also be used in this context. This will part of the future research as well as to the application of the viability theory to fault-tolerant control.

\section{REFERENCES}

[1] C. J. Tomlin, I. Mitchell, A. M. Bayen, and M. Oishi, "Computational techniques for the verification of hybrid systems," Proceedings of the IEEE, vol. 91, no. 7, pp. 986-1001, 2003.

[2] M. Chen, Q. Hu, J. F. Fisac, K. Akametalu, C. Mackin, and C. J. Tomlin, "Reachability-based safety and liveness of unmanned aerial vehicle platoons on air highways," arXiv preprint arXiv:1602.08150, 2016.

[3] S. Larosa, G. Mongardi, and F. Torielli, "An experience in formal verification of safety properties of a railway signalling control system," in Safe Comp 95: The 14th International Conference on Computer Safety, Reliability and Security, Belgirate, Italy 11-13 October 1995, p. 474, Springer Science \& Business Media, 2013.

[4] L. Larosa, A. Traverso, M. L. Ferrari, and V. Zaccaria, "Pressurized sofc hybrid systems: control system study and experimental verification," Journal of Engineering for Gas Turbines and Power, vol. 137, no. 3, p. $031602,2015$.
[5] M. Monfared, S. Golestan, and J. M. Guerrero, "Analysis, design, and experimental verification of a synchronous reference frame voltage control for single-phase inverters," iEEE Transactions on industrial Electronics, vol. 61, no. 1, pp. 258-269, 2014.

[6] T. Harris, C. Seppala, and L. Desborough, "A review of performance monitoring and assessment techniques for univariate and multivariate control systems," Journal of Process Control, vol. 9, no. 1, pp. 1-17, 1999.

[7] T. J. Harris and W. H. Ross, "Statistical process control procedures for correlated observations," The Canadian Journal of Chemical Engineering, vol. 69, no. 1, pp. 48-57, 1991.

[8] J.-P. Aubin, A. M. Bayen, and P. Saint-Pierre, Viability theory: new directions. Springer Science \& Business Media, 2011.

[9] F. Blanchini and S. Miani, Set-theoretic methods in control. Springer, 2008.

[10] P.-B. Wieber, "Viability and predictive control for safe locomotion," in Intelligent Robots and Systems, 2008. IROS 2008. IEEE/RSJ International Conference on, pp. 1103-1108, IEEE, 2008.

[11] A. M. Bayen, I. M. Mitchell, M. K. Osihi, and C. J. Tomlin, "Aircraft autolander safety analysis through optimal control-based reach set computation," Journal of Guidance, Control, and Dynamics, vol. 30, no. 1, pp. 68-77, 2007.

[12] K. Margellos and J. Lygeros, "Air traffic management with target windows: An approach using reachability," in Decision and Control, 2009 held jointly with the 2009 28th Chinese Control Conference. CDC/CCC 2009. Proceedings of the 48th IEEE Conference on, pp. 145150, IEEE, 2009.

[13] R. J. Spiteri, D. K. Pai, and U. M. Ascher, "Programming and control of robots by means of differential algebraic inequalities," Robotics and Automation, IEEE Transactions on, vol. 16, no. 2, pp. 135-145, 2000.

[14] P. Saint-Pierre, "Viable capture basin for studying differential and hybrid games: application to finance," International Game Theory Review, vol. 6, no. 01, pp. 109-136, 2004.

[15] I. M. Mitchell, "Comparing forward and backward reachability as tools for safety analysis," in International Workshop on Hybrid Systems: Computation and Control, pp. 428-443, Springer, 2007.

[16] S. Montes de Oca, V. Puig, and J. Blesa, "Robust fault detection based on adaptive threshold generation using interval lpv observers," International Journal of Adaptive Control and Signal Processing, vol. 26, no. 3, pp. 258-283, 2012.

[17] C. Combastel, "A state bounding observer based on zonotopes," in European Control Conference, 2003.

[18] C. Le Guernic and A. Girard, "Reachability analysis of linear systems using support functions," Nonlinear Analysis: Hybrid Systems, vol. 4, no. 2, pp. 250-262, 2010

[19] J. N. Maidens, S. Kaynama, I. M. Mitchell, M. M. Oishi, and G. A. Dumont, "Lagrangian methods for approximating the viability kernel in high-dimensional systems," Automatica, vol. 49, no. 7, pp. 2017-2029, 2013.

[20] M. M. Seron and J. A. De Doná, "Robust fault estimation and compensation for lpv systems under actuator and sensor faults," Automatica, vol. 52, pp. 294-301, 2015. 